\title{
Inter-loop Optimizations in RAJA using Loop Chains
}

\author{
Brandon Neth \\ University of Arizona \\ Tucson, AZ, USA \\ brandonneth@email.arizona.edu \\ Bronis R. de Supinski \\ Lawrence Livermore National Laboratory \\ Livermore, CA, USA
}

\author{
Thomas R.W. Scogland \\ Lawrence Livermore National Laboratory \\ Livermore, CA, USA
}

\author{
Michelle Mills Strout \\ University of Arizona \\ Tucson, AZ, USA
}

\begin{abstract}
Typical parallelization approaches such as OpenMP and CUDA provide constructs for parallelizing and blocking for data locality for individual loops. By focusing on each loop separately, these approaches fail to leverage sources of data locality possible due to inter-loop data reuse. The loop chain abstraction provides a framework for reasoning about and applying inter-loop optimizations. In this work, we incorporate the loop chain abstraction into RAJA, a performance portability layer for high-performance computing applications. Using the loop-chain-extended RAJA, or RAJALC, developers can have the RAJA library apply loop transformations like loop fusion and overlapped tiling while maintaining the original structure of their programs. By introducing targeted symbolic evaluation capabilities, we can collect and cache data access information required to verify loop transformations. We evaluate the performance improvement and refactoring costs of our extension. Overall, our results demonstrate $85-98 \%$ of the performance improvements of hand-optimized kernels with dramatically fewer code changes.
\end{abstract}

\section{CCS CONCEPTS}

-Software and its engineering $\rightarrow$ Software libraries and repositories.

\section{KEYWORDS}

performance portability, polyhedral analysis, data locality, symbolic execution, $\mathrm{C}++$, RAJA, loop chains

\section{ACM Reference Format:}

Brandon Neth, Thomas R.W. Scogland, Bronis R. de Supinski, and Michelle Mills Strout. 2021. Inter-loop Optimizations in RAJA using Loop Chains. In 2021 International Conference on Supercomputing (ICS '21), fune 14-17, 2021, Virtual Event, USA. ACM, New York, NY, USA, 12 pages. https://doi.org/10. $1145 / 3447818.3461665$

\footnotetext{
Permission to make digital or hard copies of all or part of this work for personal or classroom use is granted without fee provided that copies are not made or distributed for profit or commercial advantage and that copies bear this notice and the full citation on the first page. Copyrights for components of this work owned by others than ACM must be honored. Abstracting with credit is permitted. To copy otherwise, or republish, to post on servers or to redistribute to lists, requires prior specific permission and/or a fee. Request permissions from permissions@acm.org.

ICS '21, fune 14-17, 2021, Virtual Event, USA

(C) 2021 Association for Computing Machinery.

ACM ISBN 978-1-4503-8335-6/21/06 .\$15.00

https://doi.org/10.1145/3447818.3461665
}

\section{INTRODUCTION}

Scientific inquiry leverages high-performance computing (HPC) for a variety of tasks, including data analysis and experiment design. Also, experiments are often performed in silico, using simulation instead of real-world experiment. Further, the rise of machine learning gives HPC principles wider applicability than ever before.

HPC systems are incredibly diverse architecturally, both in terms of compute and data. The ten fastest HPC systems use nine different processor/coprocessor configurations [1]. This architectural diversity implies that applications designed to be performant on one system do not necessarily perform well on others. This issue of performance portability costs countless developer hours and creates a need for tools that allow developers to design a single application that performs well across systems with minimal modifications.

One such tool is the RAJA performance portability layer [24], a $\mathrm{C}++$ library. RAJA enables programmers to specify loops with templated functions, where one template parameter is an execution policy such as simd_exec, omp_parallel_for_exec, or cuda_exec. Thus the heavy lifting of porting a parallel implementation to different architectures is abstracted into standardized execution policies.

Optimizing the performance of HPC applications is more complicated than introducing new parallelism. Many applications are not compute-bound, meaning additional parallelism does not improve performance. Instead, moving data to and from memory and accelerators bottlenecks these applications. While RAJA addresses porting parallelism between systems, it lacks the ability to port inter-loop data locality optimizations. The key capability that RAJA needs to enable data locality optimizations is an inter-loop context.

When parallelizing an application, loops are generally considered and parallelized one at a time. This reduces opportunities to leverage inter-loop data reuse. The loop chain abstraction [28] captures sequences of loops that share data and enough information about how such loops access data to enable inter-loop optimization. Considering loops as a chain uncovers optimizations, like fusion and overlapped tiling, that are impossible to replicate when optimizing each loop independently. We address the problem in porting data locality optimizations in this work by incorporating loop chaining, an abstraction for balancing data locality with parallelism across loops, into RAJA. Our technique imposes a minimal code delta on the developer, leverages $\mathrm{C}++$ 's operator overloading and generic lambdas to perform necessary analysis, and requires no additional compilation steps.

This paper presents the following contributions: 
- the design of a compact RAJA extension to enable inter-loop optimizations;

- a methodology to perform symbolic evaluation in $\mathrm{C}++$ code;

- an implementation of these techniques in RAJA; and

- evaluation of their porting costs and performance benefits.

In the context of our paper, symbolic evaluation refers to the run-time process of executing loop bodies with symbolic iterators to gather data access information without affecting the state of the computation. For our RAJAPerf case study, RAJALC requires 8.5 source lines of code changed or added on average, compared to 23.625 when implementing by hand. Furthermore, in 21 of 24 benchmark-system combinations, RAJALC sees performance changes in the same direction as hand-implemented variants, and $85-98 \%$ of the performance improvement, depending on the system.

\section{THE RAJA PORTABILITY LAYER}

Before making the step to inter-loop representation and optimization, we discuss the key features of RAJA that enable performance portability as our approach leverages them. These features are an abstraction layer for data accesses and the orthogonal specification of loop schedules (i.e., separating the specification of the loop body from the loop iteration). Current usage of RAJA leverages these for loop parallelization and data layout transformations. In this paper, we use these features to build the loop chain abstraction.

\subsection{RAJA HYDRO_2D Example}

To illustrate the loop and data access abstraction and to provide context for the required coding effort, we port a loop in the HYDRO_2D benchmark from its reference $\mathrm{C} / \mathrm{C}++$ implementation to use RAJA. Listing 1 shows the before and after for the port.

RAJA has two built-in execution primitives: forall and kernel. forall is used for unnested loops, so for this example we use kernel. We must provide three pieces of information to kernel. First, we provide the execution policy, which describes the structure of the loop and what parts to run in parallel. Second, we provide a container of iterator tuples, which describe the iterator values for the loop execution. Last, we provide a lambda function that describes the loop body.

Another useful step employs RAJA's array wrapper, the View, which supports easily changing the layout of arrays. Their use is generally optional with RAJA, but is required to use our loop chain abstraction. The abstraction requires data access information, which we collect by overloading View operators. Lines 10-11 of Listing 1 show how to initialize zrout as a View object.

\subsection{Impact of RAJA}

RAJA [24] provides a methodology to improve application portability. As the high end of high performance computing transitions from traditional homogeneous core designs into heterogeneous systems, applications require a common abstraction to keep up with the variety of platforms and their associated programming models. RAJA provides that abstraction for LLNL C++ applications, which are large (i.e., a hundred thousand to low millions of lines), long lived (i.e., often 20 years or greater), and must both run and perform well on cutting-edge hardware to accomplish their goals.

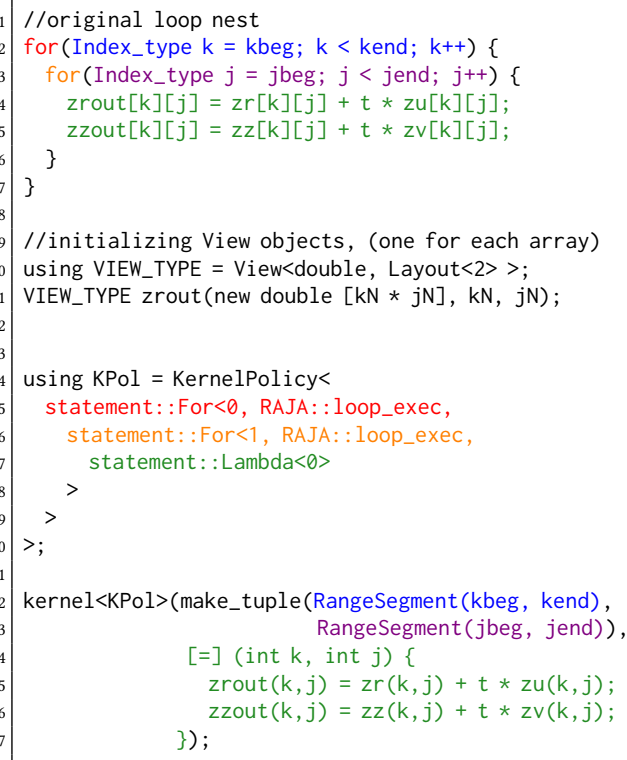

Listing 1: Hydro_2D Loop (Color indicates code regions of original and RAJA versions that perform similar functions)

RAJA has grown into a library that implements the core constructs and architecture and model-specific policies, across a myriad of platforms and backends. RAJA is in production use in eight [22] distinct LLNL applications and libraries, as well as several ECP applications including SW4 [37], GEOSX [44], and SUNDIALS [21].

Results published for three LLNL production codes [8] show performance portability across a variety of architectures. Comparing a whole node to a whole node, Ares achieved 13×, ALE3D achieved $17 \times$ and Ardra achieved $12 \times$ speedups on nodes of the LLNL Sierra system for meaningful inputs.

\section{LOOP CHAIN ABSTRACTION}

RAJA loop execution policies apply to individual loops. Often two or more loops exhibit producer/consumer data sharing. The loop chain abstraction [28] enables scheduling across such loops to improve temporal data locality. Our goal is to design RAJA techniques to express and to schedule loop chains.

\subsection{Loop Chains}

A loop chain is a sequence of loop nests $L_{0}, L_{1}, L_{2}, \ldots L_{n}$, in which each loop nest $L_{i}$ has four associated concepts:

- an iteration space $I_{i}$,

- functions $R_{i}$ and $W_{i}$ that map iteration $i$ to the data elements that it reads and writes;

- a parallelism policy $p_{i}$ that describes how the loop can be executed; and

- a loop body $B_{i}$.

The loop chain abstraction expands the possible loop scheduling scope. Using it to optimize an application consists of three stages: specifying loop objects; specifying or selecting provably correct loop transformations; and applying those transformations. 




Listing 2: Example Loop Chain

\subsection{Loop Chain Transformations}

Every loop nest has a schedule that describes the order in which the iterations should execute. A lexicographical ordering over integer vectors that represent the iterations describe this schedule. Consider the loops in Listing 2, which sum array $a$ into $b$ and then store a stencil computation on b into c.

The set $I_{0}=\{[0, i, 0, j, 0] \mid 0 \leq i<N, 0 \leq j<M\}$ describes the iterations of the first loop while the set of vectors $I_{1}=\{[1, i, 0, j, 0] \mid 1 \leq$ $i<N-1,1 \leq j<M-1\}$. describes those of the second. Within each tuple, the first element is the loop index, the second and fourth are the iteration of the loop dimensions, and the third and fifth are the statement index within the loop. We can describe the sequential loop schedules as the lexicographical ordering over $\mathbb{Z}^{5}$.

Legal loop transformations and schedules must respect the data dependences in the program [27]. These data dependences can be described as relations over the iterations of loops. Our example has dependences from the first statement, $B 0$, to the second, $B 1$, due to the writes to $b$ in the first loop and the reads from $b$ in the second. The relations $D_{i-1}=\{[0, i, 0, j, 0] \rightarrow[1, i-1,0, j, 0]\}$, $D_{i}=\{[0, i, 0, j, 0] \rightarrow[1, i, 0, j, 0]\}$, and $D_{i+1}=\{[0, i, 0, j, 0] \rightarrow[1, i+1,0, j, 0]\}$ describe those dependences. The relation induced by the read from $b[i-1][j]$ is due to the data element written in iteration $(i, j)$ of the first loop being read by iteration $(i-1, j)$ of the second loop.

A legal loop transformation maintains the dependence relations. That is, a transformation is legal if its application to the dependence relations remains a subset of the schedule relation. Consider the fusion transformation on our example loops, which maps the iteration vectors of the second loop from $[1, i, 0, j, 0]$ to $[0, i, 0, j, 1]$. Its application to the dependence relation $D_{i-1}$ yields $\{[0, i, 0, j, 0] \rightarrow[0, i-1,0, j, 1]\}$. Thus, $x>y$ in the schedule for any pair of iterations $(x, y)$ that $D_{i-1}$ relates, and so the transformation is not legal.

The loop chain abstraction uses the information associated with each loop nest to build these representations. The iteration spaces $I_{i}$ and loop bodies $B_{i}$ describe the iteration vectors; the data access functions $R_{i}$ and $W_{i}$ construct the dependence relations; and the parallelism policies $p_{i}$ inform the schedule relations.

\subsection{Loop Objects in RAJA}

Although RAJA does not currently provide chainable loop objects, it has many of the necessary components. The execution primitive and common case, kernel and forall, form the initial basis for the description of our loop nests. The first kernel argument is a collection of iterator tuples, an explicit instance of an iteration space. We can extract the parallelism policy from its execution policy, which is a template argument. The loop body is a variable-length list of lambda functions. We lack only the two data access functions, which are implicitly encoded within the loop body. Section 4.2 describes our symbolic evaluation extension that extracts them.

\section{DESIGN CHANGES TO RAJA}

We introduce an interface in RAJA for users to specify loop chains and transformations so as to enable inter-loop optimizations without additional tooling or compilation steps. We design a symbolic evaluation mechanism that gathers data access patterns of kernels. We use this mechanism to analyze kernels at runtime and use the results to select an execution schedule for the kernels.

Traditional polyhedral optimization techniques are normally broken up into identifying code regions to optimize, converting to a polyhedral representation, analysis/transformation, and code generation. Because RAJALC operates at the language level rather than the compiler level, our approach is slightly different. While identifying code regions, converting to a polyhedral representation, and analysis are roughly equivalent to compiler-level approaches, transformation and code generation are formulated differently. The fundamental difference is that transformation and code generation happen before any analysis.

\subsection{RAJA Computations as Objects}

Delayed execution is a key design pattern in embedded domainspecific languages such as Halide [40] and Tensorflow [2]. These languages separate the statements that specify a computation from those that execute it. In between, the programmer can specify how to schedule and optimize the computation. However, in standard RAJA, computational kernel statements immediately execute the specified computation. So, we introduce a wrapper class (KernelWrapper) that enables execution to be delayed until after analysis and transformation. We introduce two functions based on existing kernel execution functions to create KernelWrappers: make_forall and make_kernel. They have the same interface as their corresponding execution functions, but return a KernelWrapper object that can be executed or optimized. Listing 3 shows these wrapping functions in use.

\subsection{Gathering Access Information}

Specifying parallelism within RAJA and other programming models can build on commonly used abstractions such as forall. However, determining when inter-loop scheduling transformations such as loop fusion are legal requires an analysis of the dependences between those loops. Other loop chain approaches require specification of data access patterns by hand, which effectively rewrites the loop [10]. Our symbolic evaluation mechanism eliminates this effort for the developer by leveraging RAJA's array wrapping views abstraction.

Using RAJA Views, an array access like $a[i][j][k]$ becomes the View access $a(i, j, k)$ as Listing 4 shows. We overload the View function call operator with a symbolic iterator type to automate data access pattern collection through symbolic execution. To use our symbolic evaluation, kernel lambda arguments must be changed to auto, which allows normal execution of lambda using index values while its symbolical evaluation uses our symbolic iterators.

Figure 1 shows the process of symbolically evaluating the body of a lambda. We break kernel symbolic evaluation into two contexts: indexing and accessing. Indexing encompasses the representation and storage of index expressions, like $i+1$ in $a(i+1)$. The symbolic evaluation must retain the entire structure of the index expression because the loop's data access pattern must reflect the difference 


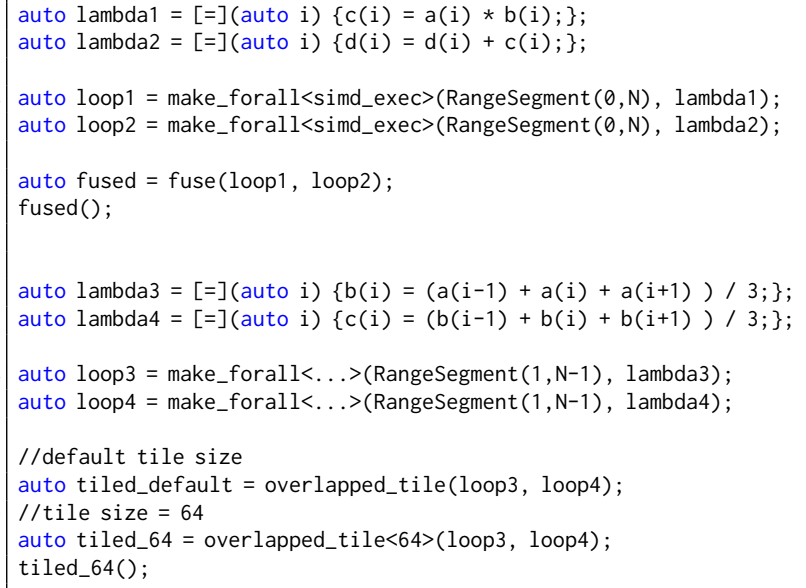

Listing 3: Using the fuse and overlapped_tile transformations.

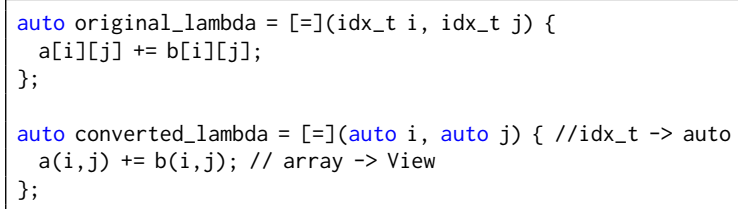

\section{Listing 4: Kernel Lambda Conversion}

between $a(i+1)$ and $a(i-1)$. Indexing evaluation is shown in steps 2 and 3 of Figure 1. Accessing encompasses the statement-level data access semantics of the kernels. Unlike with indexing, only whether accesses are reads or writes matters, not the entire structure of the statements. For example, we need to know that the statement $c(i)=a(i)+b(i)$ reads $a(i)$ and $b(i)$ and writes $c(i)$, but not that it adds $a(i)$ and $b(i)$. Access evaluation is shown in steps 4, 5, and 6 of Figure 1. Listing 5 shows the grammar for supported indexing expressions and access statements. Behavior for kernels that do not use this grammar is undefined.

Normal RAJA kernel execution only affects the states of its Views. However, symbolic evaluation should not change View states. Instead, it should only preserve the access information. We achieve this by adding a class-wide access collector to the symbolic iterator. When symbolic accesses are evaluated, they are added to the collector, and updated as reads or writes when assignments are evaluated. This access collector and its contents at steps 5 and 7 are shown to the right in Figure 1, and the change can be seen between steps 5 and 6 .

\subsection{Loop Chain Transformation Specifications}

Once we have computation objects and their access patterns, we can apply transformations. We support two transformations, fusion and overlapped tiling, each with two variants. For all transformations, the user provides the target kernel objects as arguments. Listing 3 shows two of these transformations in use.

Fusion transformations combine execution of iterations with the same iterator values to improve data locality. They work best for loops with producer-consumer relationships or those that access

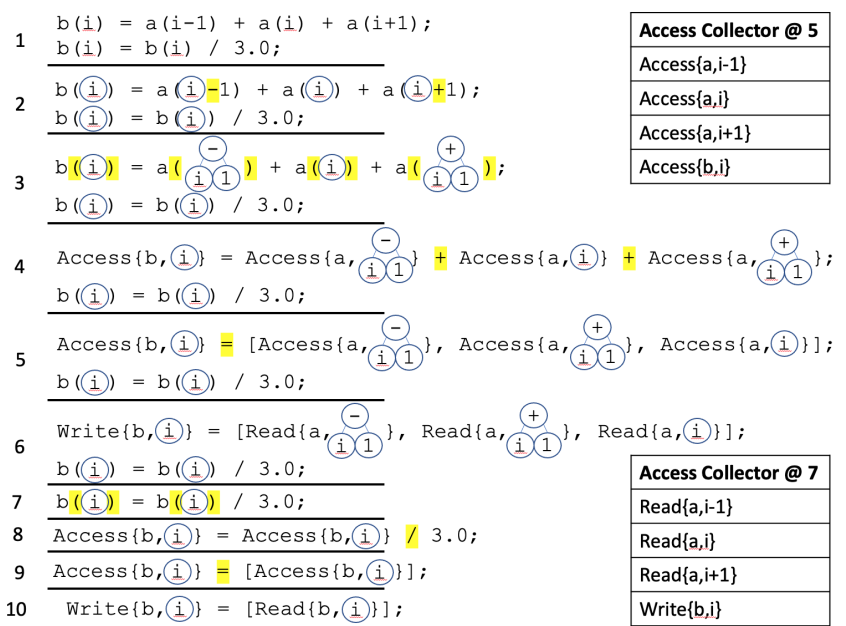

Figure 1: Symbolic evaluation of a kernel lambda. Indexing expressions are evaluated first, then accesses, then statements in terms of accesses. As assignments are evaluated, the accesses are marked as reads or writes. Accesses are tracked using the access collector.

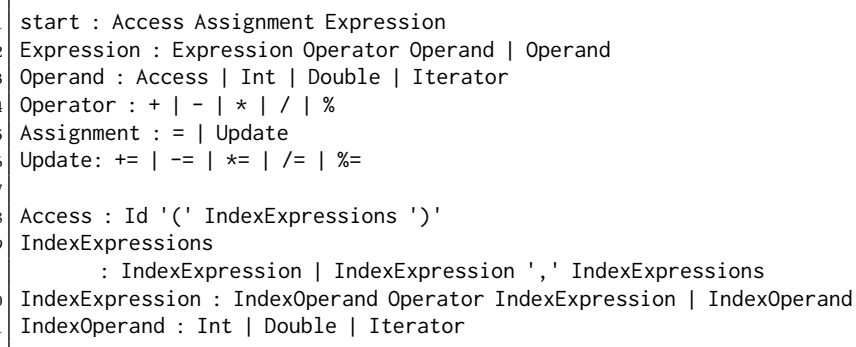

Listing 5: EBNF Grammar to Support Symbolic Evaluation

the same data. Our fusion transformations, fuse and fuse_always, support the safety and porting cost trade-off. The former uses symbolic execution to determine if the transformation is legal. The latter applies it prescriptively. If the user knows the dependences between loops do not impede fusion, it allows them to fuse kernels that do not use Views. On the other hand, fuse requires the user to use Views instead of arrays to limit the fusion transformation to when it is safe.

Overlapped tiling transformations provide improved data locality and enables parallelism by performing some redundant computation on the surface of the tiles. They work best for loops with stencil-like access patterns, where fusion results in a more restricted wavefront parallelism. Our two overlapped tiling transformations, overlapped_tile and overlapped_tile_fuse, differ in the execution schedule within each tile as Figures $2 \mathrm{~g}$ and $2 \mathrm{~h}$ show. Specifically, the schedule within the tile of overlapped_tile mimics a sequential schedule by executing all iterations of the first kernel before it executes all iterations of the second while overlapped_tile_fuse fuses individual iterations of the loops within each tile. Lines 11 through 21 of Listing 3 show how to use these transformations. 
When using the fuse variant or either overlapped tiling transformation, if analysis shows the transformation to be unsafe, a warning message is printed and the original schedule is used.

\subsection{Compile- and Run-time Actions}

When the programmer uses a RAJALC loop transformation, the compiler instantiates two different executor types for the entire loop chain. The first executor uses the transformed schedule, while the second uses the original schedule. Both executors are included with the object returned by the transformation call.

At run-time, when each kernel is initialized, its data access patterns are collected and cached. This reduces the cost of the symbolic evaluation by only performing it once, even through kernels are typically executed many times. Then, the access information is used by the transformation functions to select which schedule should be used.

\section{LOOP TRANSFORMATIONS IN RAJALC}

Section 4.3 shows how a user can specify the use of loop fusion transformations or overlapped and fusion transformations on loops in a chain. In this section, we describe how RAJALC generates different execution schedules and uses the information gathered from the symbolic evaluation to verify their correctness.

\subsection{Loop Shifting for Stencil Computations}

While fuse_always prescriptively apples a fusion transformation without checking its legality, fuse uses symbolic evaluation to guide a legal loop fusion. For a fusion to be legal, the inter-loop dependences cannot have a negative direction. However, loops can often be shifted to make such dependences non-negative. Figures $2 \mathrm{a}$ and $2 \mathrm{~b}$ show an example of shifting the loops to adjust negative dependences. Prior work showed how to convert the dependence relations into constraints of an ILP optimization problem [10]. We minimize the sum of the non-negative shift amounts $S_{1,1}, S_{1,2} \ldots, S_{2,1}, \ldots, S_{l, n}$ with the following constraints. We add constraints $S_{b, i}+d_{i} \geq S_{a, i}$ for each dependence $\left[d_{1}, d_{2}, \ldots, d_{n}\right]$ from kernel $a$ to $b$. Solutions to this problem correspond to shift amounts that enable loop fusion. We also use this shifting mechanism to simplify the dependences for overlapped tiling.

While fusion in this circumstance improves data locality, it restricts parallelism. Because fusion combines iterations of different loops, dependences that are originally between different loops become dependences between iterations of the same loop. While wavefront parallelism may still be possible, overlapped tiling often offers a better balance of parallelism and data locality [35].

\subsection{Iteration Space Alignment and Lambda Generation}

When performing optimizing transformations, kernel iteration spaces do not always completely align. Misalignments can arise from differences in the original iteration spaces or from shifts to remove negative dependences. While the iterations the loops in a chain have in common are executed by the fused or tiled kernels, we also must generate extra kernels to execute the unshared boundary iterations.

Figure 3 illustrates the generation of these kernels for one $d=3$ loop in a chain. First, we calculate the shared iteration space of the chain, using the intersection of all kernel iteration spaces, which yields a $d$-dimensional hyper-rectangle within each kernel's iteration space, as Figure $3 \mathrm{~b}$ shows. To account for the boundary iterations, we then partition the loop's iteration space, using the faces of the shared iteration space. Figures $3 \mathrm{c}$ and $3 \mathrm{~d}$ show the iteration spaces of the $2 * d=6$ boundary kernels for the $3 \mathrm{D}$ loop. This process generates $2 * d * n$ boundary kernels across the $n$ loops in a chain, plus the one fused/tiled kernel over the shared iteration space. We order these $2 * d * n+1$ kernels by loop and dimension to execute the low boundary iterations, the shared iterations, then the high boundary iterations.

Each kernel requires an iteration space and a lambda describing the loop body. For the boundary iterations, we use the original lambdas. For the shared iterations, a new lambda is generated based on the transformation. With fusion, the new lambda executes the sequence of original lambdas, performing one iteration of each loop. With overlapped tiling, the new lambda executes each original lambda across its share of the tile, as shown in Figure $2 \mathrm{~g}$.

\subsection{Overlapped Tiling}

Overlapped tiling proceeds similarly to the fuse transformation. First, we calculate and apply shifts to remove negative dependences as Figures $2 \mathrm{a}$ and $2 \mathrm{~b}$ show. We next calculate the overlapping iteration space and partition boundary iterations as Figure 2c shows. We then perform overlapped tiling as Figures $2 \mathrm{~d}-2 \mathrm{i}$ show.

Before we can generate the kernel for the overlapped tiling, we must calculate the overlap amounts. We set up an ILP optimization problem similarly to the calculation of the shift amounts. For $n$ kernels of dimension $m$, our optimization problem is over the $n m$ overlap amounts $O_{1,1}, O_{1,2}, \ldots, O_{2,1}, \ldots, O_{n, m}$. We introduce a non-negativity constraint to ensure that all overlap amounts are greater than zero. We then add the constraints $O_{a, 1}>=O_{b, 1}+d_{1}, O_{a, 2}>=O_{b, 2}+$ $d_{2}, \ldots, O_{a, m}>=O_{b, m}+d_{m}$ for each dependence $D=\left[d_{1}, d_{2}, \ldots, d_{m}\right]$ from kernel $a$ to kernel $b$, which ensures that if a tile includes an iteration of kernel $b$, it also includes all necessary iterations of kernel $a$. We then minimize the sum of the overlap amounts $\sum_{i=1}^{n} \sum_{j=1}^{m} O_{i, j}$.

After we calculate the overlap amounts, we generate a kernel that iterates over each tile, executing the original kernels for the iterations of the tile. The two overlapped tiling transformations, overlapped_tile_no_fuse and overlapped_tile_fuse differ in their schedule for executing each tile. Without fusion, we execute the tile one kernel at a time: all of $k n l 1$ is executed, then all of $k n l 2$, and so on. We constrain tile sizes for this schedule by cache size and by the size of the overlap. Tiles must be small enough to fit into cache to maximize data locality benefits, but tile size should be maximized to reduce the proportion of recomputation.

In contrast, the overlapped_tile_fuse transformation fuses iterations within each tile using the fusion algorithm described above. Introducing fusion into the tile decouples data locality from cache size. Unrestricted by cache considerations, the only limit on tile size is the need for parallelism, enabling larger tiles than otherwise possible.

\section{CASE STUDY: RAJA PERFORMANCE SUITE}

We evaluate our advances through two case studies, porting the RAJA Performance Suite (RAJAPerf) [23] and LULESH from standard RAJA to RAJALC. In both case studies, we evaluate the performance and porting process of the RAJALC implementations and we detail benefits and limitations of the approach. 


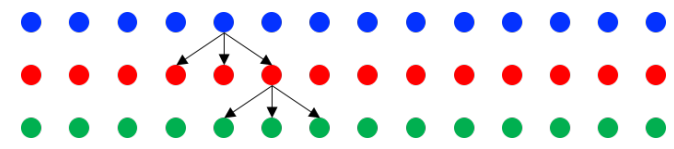

(a) Original kernel iteration spaces and (abbreviated) dependences.

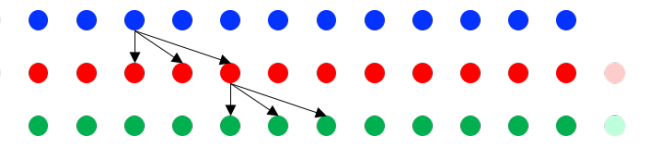

(c) Shared iteration space. Chain could be fused sequentially.

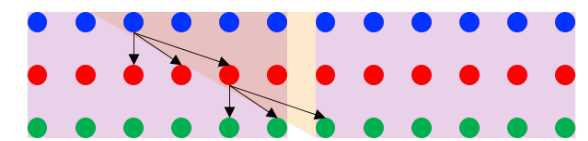

(e) Overlap for each tile shaded in orange. Tiles against low edge have no overlap.

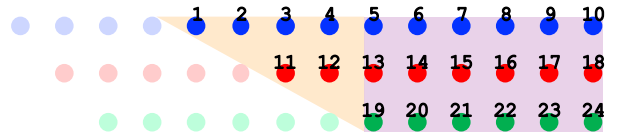

(g) Execution order for an individual tile without fusion.

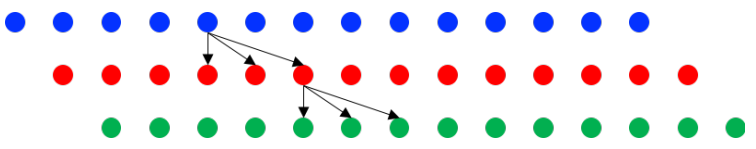

(b) After shifting kernels to remove negative dependences.

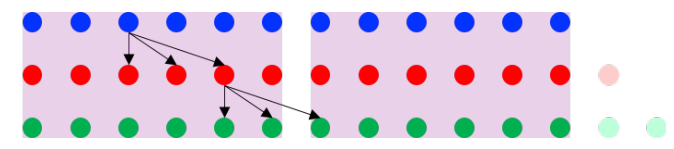

(d) Underlying tiles for TileSize $=6$ shaded in purple.

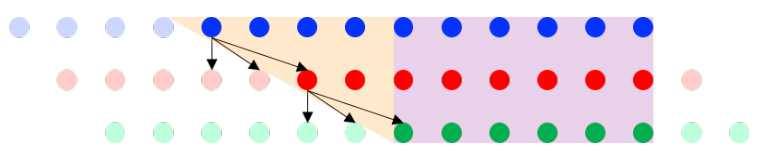

(f) An individual overlapped tile.

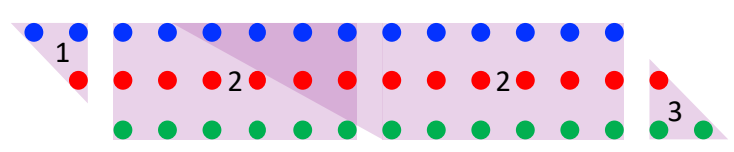

(i) Global execution order for entire computation.

Figure 2: Overlapped tiling of 3 one-dimensional loops.

The RAJA Performance Suite is a collection of benchmark kernels that are used to evaluate RAJA performance. The suite contains 46 benchmark kernels across five categories; 33 contain a single loop nest, leaving 13 kernels for which our advances are relevant. Four of these are matrix computations involving reductions to which RAJALC's current capabilities do not apply. Finally, one benchmark does not exhibit any data reuse, so no data locality optimizations are relevant, including ours. Thus, we evaluate RAJALC on 8 benchmark kernels.

For each of the 8 kernels, we identify a locality-improving transformation and implement new variants. The first variant, Hand_Opt, applies the transformations directly. The second variant, LoopChain, applies the transformation using RAJALC. We record the number of modified or added source lines of code for each variant and evaluate their performance on three systems. System Intel1 has an 8-core Intel Core i7-6900K CPU at $3.2 \mathrm{GHz}$ and $32 \mathrm{G}$ of memory. It runs Ubuntu 16.04.7 and GCC11.0. System Intel2 has a 36-core Intel Xeon E5-2695 CPU at $2.1 \mathrm{GHz}$ and $128 \mathrm{~GB}$ of memory. System Power9 has an 44-core IBM Power9 at $3.5 \mathrm{GHz}$ and $256 \mathrm{~GB}$ of memory. Systems Intel2 and Power9 run TOSS 3 and GCC8.3.1.

\subsection{Benchmark Descriptions}

We categorize each benchmark as either point-wise or stencil. Pointwise loops access at most one element of each array per iteration, while stencil loops access a fixed pattern of elements of each array per iteration. The loop bodies lambda1 and lambda2 in Listing 3 are examples of point-wise loops, while lambda3 and lambda4 are examples of stencil loops.

Three of the benchmarks, GEN_LIN_RECUR, ENERGY, and PRESSURE, are point-wise computations. GEN_LIN_RECUR comes from a general linear recurrence computation and ENERGY and PRESSURE are loops extracted from LULESH, a hydrodynamics code. For these benchmarks, loop fusion does not interfere with parallelism, so we apply that transformation.

The other five benchmarks are stencil computations: JACOBI_1D, JACOBI_2D,HEAT_3D, HYDRO_2D, and FDTD_2D.JACOBI_1D, JACOBI_2D, and HEAT_3D compute solutions to discretized PDEs in their respective dimensions. HYDRO_2D is part of an explicit hydrodynamics code. FDTD_2D is a finite-difference time-domain kernel that is unique among the stencil computations because we can fuse two of its four loops without inhibiting parallelism. Thus, we apply that transformation. The other four require shifting to be fused, so we apply overlapped tiling to retain sufficient parallelism.

\subsection{Porting}

Implementing the fusion transformation is fairly easy. For the RAJALC variant, because we can confirm their point-wise nature visually, symbolic evaluation is unnecessary. Thus, we directly apply the transformation without converting the kernels to RAJA Views. The hand-applied variant code changes are also low impact; in a 


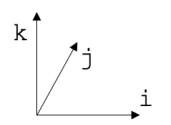

(a) Dimension order of loop.

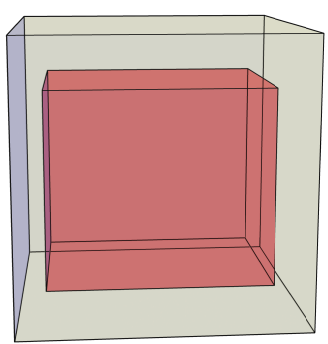

(b) Original iteration space of one loop in chain and shared iteration space executed by fused kernel.

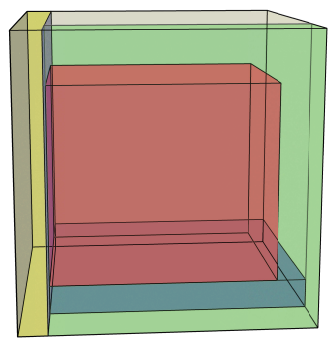

(c) Lower boundary iteration spaces.



(d) Higher boundary iteration spaces.

Figure 3: Partitioning the iteration space of a single 3d loop. The original iteration space is $(0,10) \times(0,10) \times(0,10)$ and the fused iteration space is $(1,9) \times(2,8) \times(1,8)$. This partitioning process occurs for each kernel in the chain.

real application they would not be desirable as the kernels may be used separately in other contexts. Instead of multiple calls to RAJA: : kernel with the different loop bodies, a single call is made with all loop bodies as one. For the GEN_LIN_RECUR benchmark, we also apply a loop reversal transformation by hand to both variants. RAJALC does not currently support loop reversal, but its inclusion in the implementation would not be difficult. However, automation of its use and its limited applicability in existing RAJA applications has made its implementation low priority.

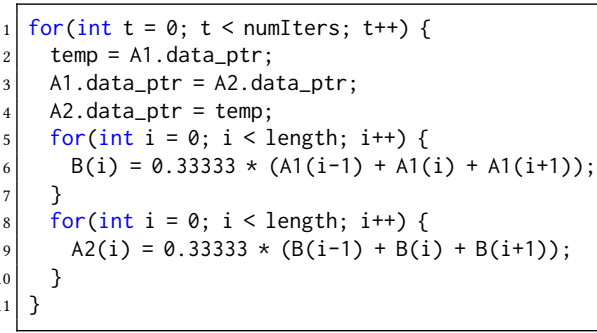

Listing 6: Double-Buffer Implementation for JACOBI_1D

For HYDRO_2D, applying overlapped tiling transformation with RAJALC requires minimal changes: we add two lines of code and change three. In contrast, the hand implementation changes four lines and added 41 new lines, effectively an order of magnitude more effort. These additional lines calculate the amounts to shift and to overlap, create the new, tiled kernel, and execute the boundary iterations not within the tiled area. These changes are not simple; the porting process encountered errors in nearly all parts: shifting, boundary iterations, tile sizes, tile bounds, and tile execution.

Porting effort in source lines of code for the other three overlapped tiling kernels is similar to HYDRO_2D, with one major difference. While HYDRO_2D has distinct input and output arrays; JACOBI_1D, JACOBI_2D, and HEAT_3D each use one of two arrays for input and output, which introduces anti-dependences that inhibit overlapped tiling. To mitigate this effect, we modify the kernels to use a doublebuffer strategy that eliminates the anti-dependence. Listing 6 shows our double-buffer reference implementation for JACOBI_1D. We add the double-buffer transformation by hand in these three variants; its automation is future work. We plan to use the access information collected during symbolic evaluation to identify chains that require it and to implement the double buffer within the View class.

Table 1 shows the number of changed and added source lines of code for all benchmarks. Overall, manual porting difficulty in source lines of code increases as the dimensionality of a kernel increases, mostly due to the specification of the boundary iterations. Alternatively, RAJALC porting difficulty is similar regardless of the loop dimensionality. Also, while with RAJALC code retains its original structure, the hand-implemented transformations obscure the underlying computation, which significantly complicates code maintenance.

\subsection{Performance}

Figure 4 shows the performance of the hand-implemented and RAJALC variants relative to the original implementations on System Intel1. For the double-buffer implementations, we show the performance relative to an un-transformed double-buffer implementation. We report the average of ten runs.

For the fused benchmarks, GEN_LIN_RECUR and FDTD_2D improve modestly while PRESSURE performance drops slightly, likely due to low amounts of data reuse. ENERGY runs more than two times faster than the original, likely due to the larger number of fused kernels than the other benchmarks. For all four, the RAJALC variant's performance is comparable to the hand-implemented loop fusion.

For the overlapped tiled benchmarks, the hand-implemented variant improves performance up to $22 \%$ for all benchmarks. The RAJALC variant, which achieves its best performance with different 


\begin{tabular}{|l|l|l|l|l|}
\hline Benchmark & \multicolumn{2}{|l|}{$\begin{array}{l}\text { Manual } \\
\text { Implementation }\end{array}$} & \multicolumn{2}{l|}{$\begin{array}{l}\text { RAJALC } \\
\text { Implementation }\end{array}$} \\
\hline & Changed & Added & Changed & Added \\
\hline ENERGY & 6 & 6 & 6 & 2 \\
PRESSURE & 2 & 3 & 2 & 2 \\
GEN_LIN_RECUR & 1 & 1 & 2 & 2 \\
FDTD_2D & 8 & 4 & 9 & 4 \\
HYDRO_2D & 4 & 41 & 3 & 2 \\
FACOBI_1D & 3 & 17 & 3 & 3 \\
FACOBI_2D & 10 & 33 & 10 & 3 \\
HEAT_3D & 12 & 38 & 12 & 3 \\
\hline Average & 5.8 & 17.9 & 5.9 & 2.6 \\
\hline
\end{tabular}

Table 1: Lines of Code Impact (Excludes double-buffer transformation changes)

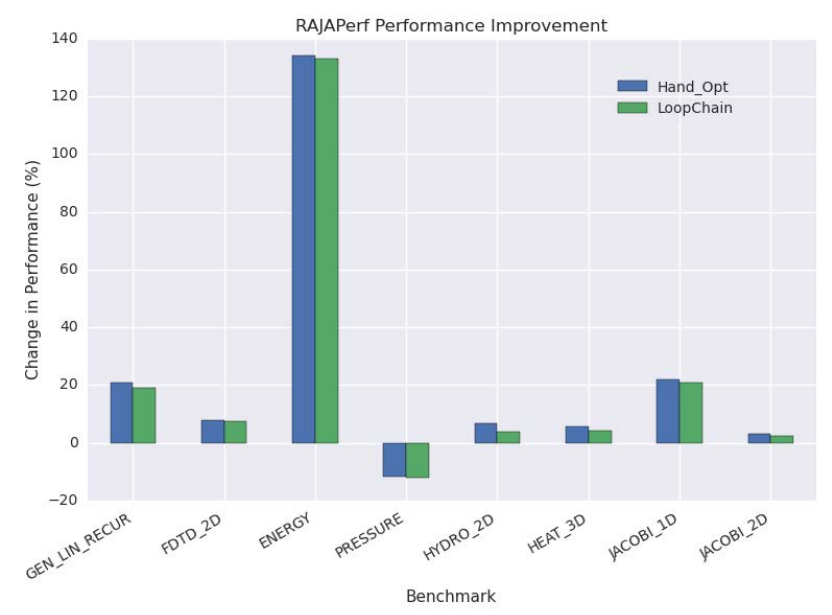

Figure 4: Performance changes of RAJAPerf benchmarks on System Intel1 (higher is better).

tile sizes, improves performance by up to $20 \%$. For both variants, the tile sizes were tuned manually. Overall, the RAJALC variant achieves $85 \%$ of the geometric mean performance improvement of the hand-implemented variant.

Because of the large thread counts on Systems Intel2 and Power9, we evaluated the scaling performance of RAJAPerf when using RAJALC. Unlike on System Intel1, RAJAPerf often did not benefit from the inter-loop optimizations on Systems Intel2 and Power9, even when applied by hand. However, the direction of RAJALC's performance impact was nearly identical to the hand-optimized code. On System Intel2, 47 of the 48 benchmark-thread-count combinations had the same direction of impact. On System Power9, all 48 combinations matched the direction of performance impact between the two variants. Overall, the geometric mean of the fraction of the handimplemented speedup achieved by RAJALC ranges across thread counts from 0.92 and 0.97 on System Intel2 and 0.95 and 1.02 on System Power9. This is evidence that RAJALC is a useful tool for quickly prototyping transformations without large refactoring costs and accurately recreates the performance impact of hand implementing the transformations.

\section{CASE STUDY: LULESH}

While our RAJAPerf case study demonstrates the types of computations RAJALC can optimize, our second case study, LULESH, explores its potential within a larger application. LULESH is a DOE proxy application for shock hydrodynamics codes. It solves a simple Sedov blast problem using the typical numerical algorithms and computational characteristics for larger codes.

As with the previous case study, we characterize the porting process and evaluate RAJALC performance compared to the original implementation. For this evaluation, we ported LULESH v2.0 [26].

\subsection{Porting}

Porting LULESH is more involved than the RAJAPerf kernels. We must first identify the part of the application to optimize,. We choose the function EvalEOSForElems, which has a long series of pointwise computations over the same iteration space. The ENERGY and PRESSURE benchmarks within RAJAPerf are from this part of LULESH.

7.1.1 Code Change: Arrays to Views. The RAJA implementation of LULESH uses RAJA execution statements, but does not use RAJA Views for arrays and vectors. Thus, we first convert these data structures to use Views. For many of the data structures, array accesses are abstracted through indexing functions. We replace these indexing functions directly with Views without changing the source code of the algorithms. However, the existing version often uses arrays, especially for local and temporary storage. We wrap these arrays with Views and change the accesses from brackets to parentheses, a tedious but simple code change.

7.1.2 Code Change: Kernel Objects. The second required change uses RAJALC kernel objects instead of direct code execution. We move the series of point-wise computations over the same iteration space in EvalEOSForElems into many individual functions. We modify them to create and to return the kernel objects, which allows us to fuse them, both by hand and with RAJALC.

7.1.3 Challenge: Indirect Accesses. The nodel ist function, which creates a list of indices for the area around an element, poses a challenge to our LULESH porting. Because this function takes the loop index and returns a list of indices, it interferes with symbolic evaluation. Future work will support symbolic evaluation for indirect accesses, with the goal of transforming sparse matrix and sparse tensor codes, where indirect accesses are especially common. RAJALC has similarities to inspector-executor strategies commonly used when optimizing sparse codes, which may provide some direction in attempts to optimize RAJA-based sparse codes.

7.1.4 Challenge: Non-contiguous Iteration Spaces. LULESH is an unstructured application: its iteration spaces are collections of ranges instead of contiguous ranges. For example, instead of iterating over every value from 0 to 100 , it may iterate over values 0 to 10 , then 17 to 23 , then 52,73 , and 75 to 100 . RAJALC currently only handles contiguous iteration spaces, so it only applies fusion to LULESH problems that use contiguous ranges. In practice, this limitation is a 


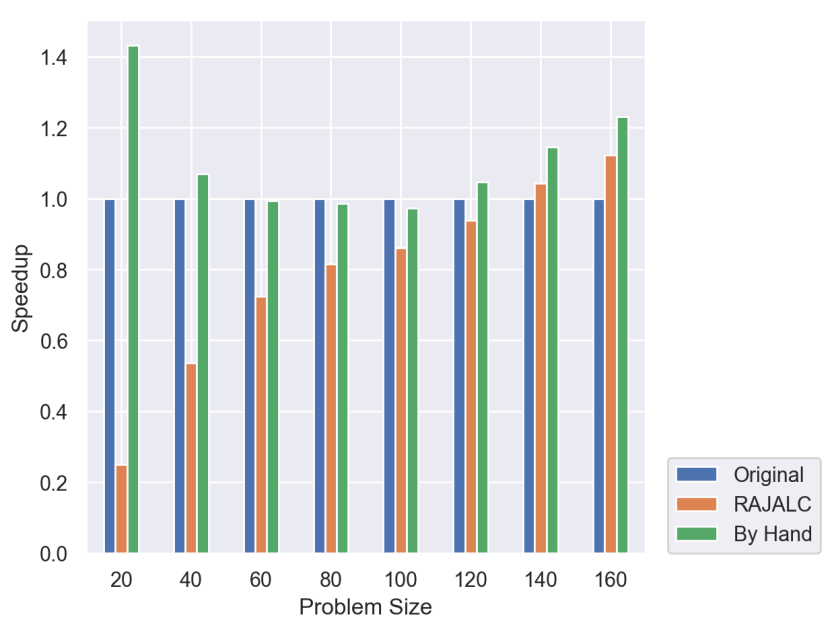

Figure 5: LULESH Speedup on System Intel1 (higher is better).

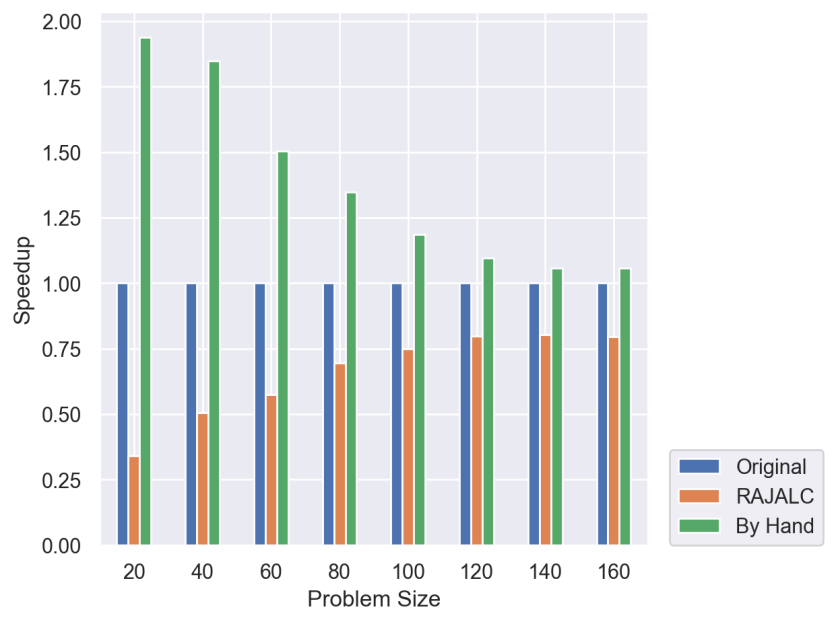

Figure 6: LULESH Speedup on System Intel2 (higher is better).

benefit over hand optimizations that assume such ranges. Nonetheless, the prevalence of unstructured scientific applications makes fusion of unstructured ranges a valuable future research direction.

\subsection{Performance}

We evaluate the performance of three LULESH variants: Original, RAJALC, and By Hand. Original is the original v2 RAJA implementation. RAJALC is the version ported to apply loop fusion with RAJALC while By Hand directly applies loop fusion by hand.

Figures 5, 6, and 7 show the average speedup over three runs relative to Original for several problem sizes. For the recommended problem sizes on Systems Intel1 and Intel2, RAJALC does not see performance improvements, but By Hand does. We expect improvement since the fused kernels are the same as ENERGY and PRESSURE in RAJAPerf. We examine the generated code with OptVis [14], to uncover two likely culprits. First, although the RAJALC code includes

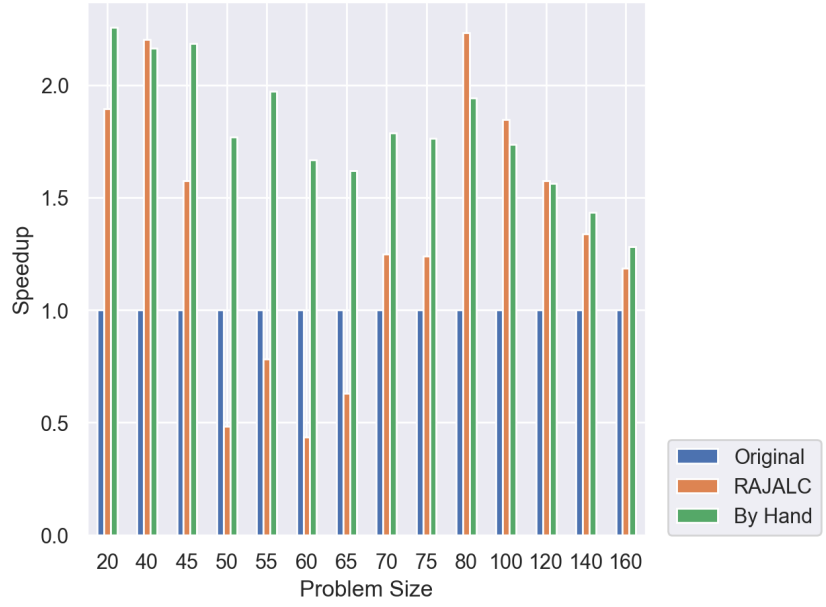

Figure 7: LULESH Speedup on System Power9 (higher is better).

inlining directives, some calls within the fused kernel were not inlined. In the By Hand variant, the calls are always inlined by virtue of the code being combined into a single lambda. Introducing multiple extra calls each iteration of the fused loop likely contributes to the performance degradation with RAJALC. Second, the code for the kernels that were successfully inlined is dispersed throughout the binary and contains more than twice as many basic blocks as the By Hand variant. These extra jumps, often to completely different parts of the binary, also likely reduce RAJALC performance. One method to reduce the first effect has been addressed by the authors in an OpenMP feature proposal. Modern compiler inlining directives are generally only suggestions to the compiler. A standardized, forced inlining directive would reduce the problem of extra calls within the kernel. However, our implementation makes use of forced inlining directives, so future work will examine why these directives are not leading to the expected binary structure.

System Power9 exhibits much larger performance improvements, including at small problem sizes. However, there is anomalous results for RAJALC for problem sizes around 60. We include additional problem sizes for System Power9 to contextualize this drop in performance. While not as significant, there is also volatility in the hand-implementation performance for these problem sizes.

\section{RELATED WORK}

Several other approaches provide performance-portable loop optimization. Inter-loop optimization has been studied for decades, but usually within the context of domain-specific languages (DSLs). Our work aims to enable more general approaches.

\subsection{Portable Intra-Loop Optimization}

RAJA [24], OpenMP [36], Kokkos [16], and SYCL [18] provide ways to parallelize and provide data locality within individual loops. For performance portability, Pennycook et al. [38] show an example that uses OpenMP to have a single source code with specializations in pragmas to target different architectures. 
Active libraries [55] such as the Matrix Template Library [45], Blitz++ [54], and the Bernoulli compiler $[4,46]$ fuse some computations using expression templates, but cannot fuse across statements.

\subsection{By Hand Inter-Loop Optimization}

Some work has experimented with overlapped tiling and other interloop scheduling by hand. Olschanowsky et al. [35] showed that scheduling across loops and reducing the temporary storage requirements led to problem size scaling and significantly better performance in a CFD application. Wahib and Maruyama [56] showed the importance of fusing kernel computations for GPU execution.

Numerous approaches to scheduling across an outer "time loop" in a stencil code have been investigated as early as 1998 [7, 57]. The Cactus project [5,42] and Ding and $\mathrm{He}[15]$ demonstrated overlapped tiling by expanding ghost cells in stencil computations. Wonnacott and Strout [58] review many other approaches to time tiling for PDE codes that do explicit stepping versus implicit stepping.

\subsection{DSLs Providing Inter-Loop Optimization}

A number of DSLs have been developed for specifying and optimizing stencil computations. The Pochoir work [49] embedded cache oblivious performance optimizations to improve temporal locality in C++ code. STELLA [19] and YASK [60] can specify and tune 3D finite difference stencil computations. Rawat et al. [41] present a DSL called StencilGen for specifying stencil computations. They present algorithms that perform overlapped tiling within stencil computations and heuristics for fusing between the computations for GPUs. Their work demonstrates some possible performance benefits of scheduling across stencil computations when targeting GPUs and CPUs. In contrast, we present ways to enable and to control these optimizations across loops in the context of a more general, parallel library, specifically RAJA.

LIFT [20] is a data parallel, functional intermediate representation that supports dense computations and stencil computations. Once a computation written in a DSL is transformed to LIFT, pattern-based transformation can implement optimizations such as overlapped tiling and target many different architectures. Krishnamoorthy et al. [29] present an automated tiling technique for stencil computations in which neighboring tiles perform overlapping computations, which reduces communication and improves load balance.

Embedded DSLs for image processing pipelines, such as Halide [39, 40] and PolyMage [34], support orthogonally specifying and scheduling stencil computations. By exposing separate mechanisms for scheduling them, PolyMage supports various scheduling, fusion, and tile size selection algorithms [3, 25, 33]. However, using these embedded DSLs limits the expressible computation and requires prohibitive porting costs for large applications.

Several DSLs that specify deep learning neural network architectures, including Halide extended for reverse mode automatic differentiation [30], Tensor Comprehensions [53], TVM [12] and Latte [52] optimize across loops. These DSLs have specific approaches to scheduling such as the Halide scheduling algorithm for DNN [59]. AutoTVM in TVM [13] learns how to schedule. Frameworks like Delite [48] for developing DSLs support cross-computation scheduling.

Although the DSL approach can better target a particular application domain, our work to schedule across loops within RAJA supports a broader class of applications. Further, we provide a different separation of concerns. RAJA handles performance portability while our loop chain extension handles scheduling across loops. DSL approaches handle all of the scheduling and targeting of architectures, which leads to not all architectures being covered in some cases.

\subsection{Traditional Compiler Approaches}

Loop optimizations are used in a wide variety of compilers. Polyhedral optimizations have been incorporated into production compilers with Graphite [51] and Polly [17]. Both works have similar approaches, starting by extracting polyhedral representation from an intermediate representation. Graphite works on the GNU GIMPLE representation and Polly on LLVM-IR. After extracting polyhedral representations, they perform a number of analyses and transformations and regenerate new, optimized code. Both approaches are general-purpose, automatic approaches.

PLuTo [11] is another example of an automatic optimizing compiler. While Graphite and Polly perform optimizations as they lower from code to executable, PLuTo is a source-to-source optimizer. While it focuses on balancing parallelism and locality through loop tiling, PLuTo also has the ability to fuse producer-consumer loops. PLuTo searches a large space of possible tilings, but it does so automatically, leaving minimal room for the developer to select their own transformations.

Other approaches like AlphaZ [61] and CHiLL [50] provide scripting languages that iteratively transform loops in the program. The programmer writes an additional file containing the transformations they want applied to loops in the program such as permute, tile, or unroll. While these approaches allow more flexibility in transformation and maintain the original structure of their code, they introduce an additional stage in the toolchain. A similar drawback applies to embedded DSLs that use their own compilers, such as Tiramisu [6].

\subsection{Staging and Other Loop Chain Approaches}

The most related work to this submission is work that more generally gathers a computational representation and other work that aims to specify and optimize loop chains. Lightweight modular staging [43] presented an approach for gathering a representation of computation to enable scheduling across that implementation in Scala.

Bertolacci et al. $[9,10]$ propose extensions to OpenMP pragmas to express and schedule loop chains. The approach requires annotations about data accesses in the pragmas. That work enables the specification of loop fusion and wavefront tiling.

Luporini et al. [31] has a similar goal of enabling the source code that uses a library (in this case Devito [32]) to schedule across loop computations that share data. The loops in their case have indirect array accesses. Thus grouping computations to improve data locality requires a run-time inspection phase [47]. Luporini et al. use various Python library interfaces to specify computations using per-loop modularization and then a lazy-computation step that gathers the loop descriptions and generates inspector-executor code for performing sparse tiling. The key differences between their work and ours is that we leverage the view capability of RAJA to avoid needing to annotate how data is being accessed in each loop. Instead, our symbolic analysis collects that information. 


\section{CONCLUSION}

Inter-loop optimizations have long been known to improve data locality and to reduce synchronization costs of computations with loops the share data with producer-consumer relationships. However, the required refactoring has remained a tedious manual process with significant impact on code maintainability. We have presented a design that retains the loop-based modularization typical of scientific codes while enabling the automated application of important inter-loop optimizations. RAJALC adds a loop chain construct and some API parameters to enable symbolic analysis of data access patterns in the RAJA performance-portability $\mathrm{C}++$ library. Importantly their use entails minimal code changes. Future research directions will address transformations for non-stencil computations and imperfectly nested loops, code coverage for symbolic execution, and automatic tuning of transformation parameters like tile size.

\section{REFERENCES}

[1] Top500 list - june 2020, June 2020

[2] M. Abadi, P. Barham, J. Chen, Z. Chen, A. Davis, J. Dean, M. Devin, S. Ghemawat, G. Irving, M. Isard, M. Kudlur, J. Levenberg, R. Monga, S. Moore, D. G. Murray, B. Steiner, P. Tucker, V. Vasudevan, P. Warden, M. Wicke, Y. Yu, and X. Zheng. Tensorflow: A system for large-scale machine learning. In Proceedings of the 12th USENIX Conference on Operating Systems Design and Implementation, OSDI'16, page 265?283, USA, 2016. USENIX Association.

[3] A. Adams, K. Ma, L. Anderson, R. Baghdadi, T.-M. Li, M. Gharbi, B. Steiner, S. Johnson, K. Fatahalian, F. Durand, and J. Ragan-Kelley. Learning to optimize halide with tree search and random programs. ACM Trans. Graph., 38(4), July 2019

[4] N. Ahmed, N. Mateev, and K. Pingali. A framework for sparse matrix code synthesis from high-level specifications. In Proceedings of the 2000 ACM/IEEE conference on Supercomputing (CDROM), Supercomputing '00, Washington, DC, USA, 2000. IEEE Computer Society.

[5] G. Allen, T. Dramlitsch, I. Foster, T. Goodale, N. Karonis, M. Ripeanu, E. Seidel, and B. Toonen. Cactus-g toolkit: Supporting efficient execution in heterogeneous distributed computing environments. In In Proceedings of 4th Globus Retreat, 2000

[6] R. Baghdadi, J. Ray, M. B. Romdhane, E. Del Sozzo, A. Akkas, Y. Zhang, P. Suriana S. Kamil, and S. Amarasinghe. Tiramisu: A polyhedral compiler for expressing fast and portable code. In 2019 IEEE/ACM International Symposium on Code Generation and Optimization (CGO), pages 193-205. IEEE, 2019

[7] F. Bassetti, K. Davis, and D. Quinlan. Optimizing transformations of stencil operations for parallel object-oriented scientific frameworks on cache-based architectures. Lecture Notes in Computer Science, 1505, 1998.

[8] D. A. Beckingsale, J. Burmark, R. Hornung, H. Jones, W. Killian, A. J. Kunen, O. Pearce, P. Robinson, B. S. Ryujin, and T. R. Scogland. Raja: Portable performance for large-scale scientific applications. In 2019 IEEE/ACM International Workshop on Performance, Portability and Productivity in HPC (P3HPC), nov 2019.

[9] I. J. Bertolacci, M. M. Strout, S. Guzik, J. Riley, and C. Olschanowsky. Identifying and scheduling loop chains using directives. In Proceedings of the Third International Workshop on Accelerator Programming Using Directives, WACCPD '16, pages 57-67, Piscataway, NJ, USA, 2016. IEEE Press.

[10] I. J. Bertolacci, M. M. Strout, J. Riley, S. Guzik, E. C. Davis, and C. Olschanowsky Using the loop chain abstraction to schedule across loops in existing code. International fournal of High Performance Computing and Networking, 13(1), January 2019.

[11] U. Bondhugula, A. Hartono, J. Ramanujam, and P. Sadayappan. Pluto: A practical and fully automatic polyhedral program optimization system. In Proceedings of the ACM SIGPLAN 2008 Conference on Programming Language Design and Implementation (PLDI 08), Tucson, AZ (fune 2008). Citeseer, 2008.

[12] T. Chen, T. Moreau, Z. Jiang, L. Zheng, E. Yan, H. Shen, M. Cowan, L. Wang, Y. Hu, L. Ceze, C. Guestrin, and A. Krishnamurthy. TVM: An automated end-to-end optimizing compiler for deep learning. In 13th USENIX Symposium on Operating Systems Design and Implementation (OSDI 18), pages 578-594, Carlsbad, CA, Oct. 2018. USENIX Association.

[13] T. Chen, L. Zheng, E. Yan, Z. Jiang, T. Moreau, L. Ceze, C. Guestrin, and A. Krishnamurthy. Learning to optimize tensor programs. Technical report, arXiv:1805.08166v4, 8 January 2019.

[14] S. Devkota, P. Aschwanden, A. Kunen, M. Legendre, and K. E. Isaacs. Ccnav: Understanding compiler optimizations in binary code. IEEE transactions on visualization and computer graphics, 2020.

[15] C. Ding and Y. He. A ghost cell expansion method for reducing communications in solving pde problems. In Proceedings of the ACM/IEEE Conference on Supercomputing, Supercomputing '01, pages 50-50, New York, NY, USA, 2001. ACM.
[16] H. C. Edwards, C. R. Trott, and D. Sunderland. Kokkos: Enabling manycore performance portability through polymorphic memory access patterns. Fournal of Parallel and Distributed Computing, 74(12):3202 - 3216, 2014.

[17] T. Grosser, H. Zheng, R. Aloor, A. Simbürger, A. Größlinger, and L.-N. Pouchet. Polly-polyhedral optimization in llvm. In Proceedings of the First International Workshop on Polyhedral Compilation Techniques (IMPACT), volume 2011, page 1, 2011.

[18] K. O. W. Group. Sycl specification, version 1.2.1 revision 6. Technical report, The Knronos Group, November 2019.

[19] T. Gysi, C. Osuna, O. Fuhrer, M. Bianco, and T. C. Schulthess. Stella: A domainspecific tool for structured grid methods in weather and climate models. In Proceedings of the International Conference for High Performance Computing, Networking, Storage and Analysis, SC '15, pages 41:1-41:12, New York, NY, USA, 2015. ACM.

[20] B. Hagedorn, L. Stoltzfus, M. Steuwer, S. Gorlatch, and C. Dubach. High performance stencil code generation with lift. In Proceedings of the 2018 International Symposium on Code Generation and Optimization, CGO 2018, pages 100-112, New York, NY, USA, 2018. Association for Computing Machinery.

[21] A. C. Hindmarsh, P. N. Brown, K. E. Grant, S. L. Lee, R. Serban, D. E. Shumaker, and C.S. Woodward. SUNDIALS: Suite of nonlinear and differential/algebraic equation solvers. ACM Transactions on Mathematical Software (TOMS), 31(3):363-396, 2005.

[22] R. D. Hornung and D. A. Beckingsale. ECP ST Project 2.3.1.06-STPM08-RAJA. Technical report, Lawrence Livermore National Lab.(LLNL), Livermore, CA (United States), 2019.

[23] R. D. Hornung, H. E. Hones, et al. Raja performance suite. Technical report, Lawrence Livermore National Lab.(LLNL), Livermore, CA (United States), 2017.

[24] R. D. Hornung and J. A. Keasler. The RAJA portability layer: Overview and status. Technical Report Tech. Rep. LLNL-TR-661403, Lawrence Livermore National Laboratory, September 2014.

[25] A. Jangda and U. Bondhugula. An effective fusion and tile size model for optimizing image processing pipelines. In Proceedings of the 23rd ACM SIGPLAN Symposium on Principles and Practice of Parallel Programming, PPoPP '18, pages 261-275, New York, NY, USA, 2018. Association for Computing Machinery.

[26] I. Karlin, J. Keasler, and R. Neely. Lulesh 2.0 updates and changes. Technical Report LLNL-TR-641973, August 2013.

[27] W. Kelly and W. Pugh. A unifying framework for iteration reordering transformations. In IEEE First International Conference on Algorithms and Architectures for Parallel Processing (ICAPP), volume 1, pages 153-162, 1995.

[28] C. D. Krieger, M. M. Strout, C. Olschanowsky, A. Stone, S. Guzik, X. Gao, C. Bertolli, P. H. J. Kelly, G. Mudalige, B. Van Straalen, and S. Williams. Loop chaining: A programming abstraction for balancing locality and parallelism. In 2013 IEEE International Symposium on Parallel Distributed Processing, Workshops and Phd Forum, pages 375-384, May 2013.

[29] S. Krishnamoorthy, M. Baskaran, U. Bondhugula, J. Ramanujam, A. Rountev, and P. Sadayappan. Effective automatic parallelization of stencil computations. In Proceedings of Programming Languages Design and Implementation (PLDI), volume 42, pages 235-244, New York, NY, USA, 2007. ACM.

[30] T.-M. Li, M. Gharbi, A. Adams, F. Durand, and J. Ragan-Kelley. Differentiable programming for image processing and deep learning in halide. ACM Trans. Graph., 37(4):139:1-139:13, July 2018.

[31] F. Luporini, M. Lange, C. T. Jacobs, G. J. Gorman, J. Ramanujam, and P. H. J. Kelly. Automated tiling of unstructured mesh computations with application to seismological modeling. ACM Trans. Math. Softw., 45(2), May 2019.

[32] F. Luporini, M. Lange, M. Louboutin, N. Kukreja, J. Hückelheim, C. Yount, P. A. Witte,P.H.J.Kelly, G.J. Gorman, and F.J. Herrmann. Architecture and performance of devito, a system for automated stencil computation. CoRR, abs/1807.03032, 2018.

[33] R. T. Mullapudi, A. Adams, D. Sharlet, J. Ragan-Kelley, and K. Fatahalian. Automatically scheduling halide image processing pipelines. ACM Trans. Graph., 35(4):83:1-83:11, July 2016.

[34] R. T. Mullapudi, V. Vasista, and U. Bondhugula. Polymage: Automatic optimization for image processing pipelines. In Proceedings of the Twentieth International Conference on Architectural Support for Programming Languages and Operating Systems, ASPLOS '15, pages 429-443, New York, NY, USA, 2015. ACM.

[35] C. Olschanowsky, M. M. Strout, S. Guzik, J. Loffeld, and J. Hittinger. A study on balancing parallelism, data locality, and recomputation in existing pde solvers. In In The IEEE/ACM International Conference for High Performance Computing, Networking, Storage and Analysis (SC), November 2014.

[36] OpenMP Architecture Review Board. OpenMP application programming interface, version 5.0. Technical Report November, Available: https://www.openmp.org/wpcontent/uploads/OpenMP-API-Specification-5.0.pdf, 2018.

[37] R. Pankajakshan, P. Lin, and B. Sjögreen. Porting a 3d seismic modeling code (SW4) to CORAL machines. IBM fournal of Research and Development, 64(3/4):17:1-17:11, 2020.

[38] S. J. Pennycook, J. D. Sewall, and J. R. Hammond. Evaluating the impact of proposed openmp 5.0 features on performance, portability and productivity. In 2018 IEEE/ACM International Workshop on Performance, Portability and Productivity in HPC (P3HPC), pages 37-46, Nov 2018.

[39] J. Ragan-Kelley, A. Adams, S. Paris, M. Levoy, S. Amarasinghe, and F. Durand. Decoupling algorithms from schedules for easy optimization of image processing pipelines. ACM Trans. Graph., 31(4):32:1-32:12, July 2012. 
[40] J. Ragan-Kelley, C. Barnes, A. Adams, S. Paris, F. Durand, and S. Amarasinghe Halide: a language and compiler for optimizing parallelism, locality, and recomputation in image processing pipelines. In Proceedings of the 34th ACM SIGPLAN conference on Programming language design and implementation, PLDI '13, pages 519-530, New York, NY, USA, 2013. ACM.

[41] P. S. Rawat, M. Vaidya, A. Sukumaran-Rajam, M. Ravishankar, V. Grover A. Rountev, L. Pouchet, and P. Sadayappan. Domain-specific optimization and generation of high-performance gpu code for stencil computations. Proceedings of the IEEE, 106(11):1902-1920, Nov 2018.

[42] M. Ripeanu, A. Iamnitchi, and I. T. Foster. Cactus application: Performance predictions in grid environments. In Proceedings of the 7th International Euro-Par Conference Manchester on Parallel Processing, Euro-Par '01, pages 807-816, London, UK, UK, 2001. Springer-Verlag.

[43] T. Rompf and M. Odersky. Lightweight modular staging: A pragmatic approach to runtime code generation and compiled dsls. Communications of the ACM, 55(6):121-130, 2012.

[44] R. R. Settgast, J. White, B. Corbett, A. Vargas, C. Sherman, P. Fu, and C. Annavarapu Geosx simulation framework. Technical report, Lawrence Livermore National Lab.(LLNL), Livermore, CA (United States), 2018.

[45] J. G. Siek and A. Lumsdaine. The matrix template library: Generic components for high-performance scientific computing. Computing in Science and Engineering, 1(6):70-78, Nov 1999.

[46] P. V. Stodghill. A relational approach to the automatic generation of sequential sparse matrix codes. PhD thesis, Ithaca, NY, USA, 1997.

[47] M. M. Strout, F. Luporini, C. D. Krieger, C. Bertolli, G.-T. Bercea, C. Olschanowsky, J. . Ramanujam, and P. H. Kelly. Generalizing run-time tiling with the loop chain abstraction. In In 28th IEEE International Parallel and Distributed Processing Symposium (IPDPS), May 2014.

[48] A. K. Sujeeth, K. J. Brown, H. Lee, T. Rompf, H. Chafi, M. Odersky, and K. Olukotun Delite: A compiler architecture for performance-oriented embedded domainspecific languages. ACM Trans. Embed. Comput. Syst., 13(4s):134:1-134:25, Apr 2014.

[49] Y. Tang, R. A. Chowdhury, B. C. Kuszmaul, C.-K. Luk, and C. E. Leiserson. The pochoir stencil compiler. In Proceedings of the 23rd ACM Symposium on Parallelism in Algorithms and Architectures, SPAA '11, pages 117-128, New York, NY, USA 2011. ACM.

[50] A. Tiwari, C. Chen, J. Chame, M. Hall, and J. K. Hollingsworth. A scalable auto-tuning framework for compiler optimization. In 2009 IEEE International Symposium on Parallel \& Distributed Processing, pages 1-12. IEEE, 2009.

[51] K. Trifunovic, A. Cohen, D. Edelsohn, F. Li, T. Grosser, H. Jagasia, R. Ladelsky, S. Pop, J. Sjödin, and R. Upadrasta. Graphite two years after: First lessons learned from real-world polyhedral compilation. In GCC Research Opportunities Workshop (GROW'10), 2010.

[52] L. Truong, R. Barik, E. Totoni, H. Liu, C. Markley, A. Fox, and T. Shpeisman. Latte: A language, compiler, and runtime for elegant and efficient deep neural networks. In Proceedings of the 37th ACM SIGPLAN Conference on Programming Language Design and Implementation, PLDI '16, pages 209-223, New York, NY, USA, 2016. ACM

[53] N. Vasilache, O. Zinenko, T. Theodoridis, P. Goyal, Z. DeVito, W. S. Moses, S. Verdoolaege, A. Adams, and A. Cohen. Tensor comprehensions: Framework-agnostic high-performance machine learning abstractions. Technical report, arXiv: 1802.04730, 2018.

[54] T. L. Veldhuizen. Blitz++: The library that thinks it is a compiler. In H. Langtangen, A. Bruaset, and E. Quak, editors, Advances in Software Tools for Scientific Computing, volume 10, pages 57-87. Springer Berlin Heidelberg, 2000.

[55] T. L. Veldhuizen and D. Gannon. Active libraries: Rethinking the roles of compilers and libraries. In Proceedings of the SIAM Workshop on Object Oriented Methods for Inter-operable Scientific and Engineering Computing (OO'98). SIAM Press, 1998.

[56] M. Wahib and N. Maruyama. Scalable kernel fusion for memory-bound gpu applications. In SC '14: Proceedings of the International Conference for High Performance Computing, Networking, Storage and Analysis, pages 191-202, 2014.

[57] D. Wonnacott. Using time skewing to eliminate idle time due to memory bandwidth and network limitations. In Proceedings of the 14th International Symposium on Parallel and Distributed Processing, IPDPS '00, Washington, DC, USA, 2000. IEEE Computer Society.

[58] D. G. Wonnacott and M. M. Strout. On the scalability of loop tiling techniques. In Proceedings of the 3rd International Workshop on Polyhedral Compilation Techniques (IMPACT), January 2013.

[59] X. Yang, M. Gao, Q. Liu, J. Setter, J. Pu, A. Nayak, S. Bell, K. Cao, H. Ha, P. Raina, C. Kozyrakis, and M. Horowitz. Interstellar: Using halide's scheduling language to analyze dnn accelerators. In Proceedings of the Twenty-Fifth International Conference on Architectural Support for Programming Languages and Operating Systems, ASPLOS '20, pages 369-383, New York, NY, USA, 2020. Association for Computing Machinery.

[60] C. Yount, J. Tobin, A. Breuer, and A. Duran. Yask-yet another stencil kernel: A framework for hpc stencil code-generation and tuning. In Proceedings of the Sixth International Workshop on Domain-Specific Languages and High-Level Frameworks for HPC, WOLFHPC '16, pages 30-39. IEEE Press, 2016.
[61] T. Yuki, G. Gupta, D. Kim, T. Pathan, and S. Rajopadhye. Alphaz: A system for design space exploration in the polyhedral model. In International Workshop on Languages and Compilers for Parallel Computing, pages 17-31. Springer, 2012. 